\title{
Nuevas pedagogías artivistas para la educación: evidencias de la construcción de convivencias democráticas y aprendizajes cooperativos en contextos vulnerables
}

\section{New artivist pedagogies for education: evidence of the construction of democratic coexistence and cooperative learning in vulnerable contexts}

Jhon Holguin-Alvarez, ${ }^{1 a}$ Aquila Priscila Montañez Huancaya, ${ }^{2}$ Norma Nancy Montañez Huancaya, ${ }^{3}$ Rosa Guillermina Dolorier Zapata, ${ }^{4}$ Juana María Cruz Montero ${ }^{5}$,Fernando Eli Ledesma Pérez ${ }^{6}$

Universidad César Vallejo, Lima, Perú156

Universidad Nacional de Educación Enrique Guzmán y Valle, Lima, Perú234

(iD) Orcid: https://orcid.org/0000-0001-5786-0763 ${ }^{1}$

(iD) Orcid: https://orcid.org/0000-0003-2754-1520²

(iD) Orcid: https://orcid.org/0000-0003-2944-86533

(iD) Orcid: https://orcid.org/0000-0003-3345-00274

(iD) Orcid: https://orcid.org/0000-0002-7772-66815

(iD) Orcid: https://orcid.org/0000-0003-4572-13816

Recibido: 03 de enero de 2020

Aceptado: 25 de abril de 2020

\begin{abstract}
Resumen
El objetivo del estudio es comprobar los efectos de pedagogías artivistas en la convive ncia democrática y los aprendizajes cooperativos en escolares de contextos vulnerables. El enfoque fue cuantitativo, de diseño experimental. Se estructuraron dos experimentos con muestras experimentales de: a) 85 estudiantes de cuarto a sexto grado de educación primaria, b) 65 de sexto grado de educación primaria. Se halló que: 1) la pedagogía artivista popular basada en aspectos culturales (problemas y folklore) desarrolla la convivencia democrática en contextos vulnerables, y 2) la pedagogía basada en dáctilo pintura teatral desarrolla los aprendizajes cooperativos de forma significativa y sus dimensiones, aunque la interacción individual es un aspecto complejo de desarrollar. Por tanto, se determinó que la pedagogía artivista popular y la experiencia de dáctilo pintura teatral son pedagogías útiles para el desarrollo de la democracia y la cooperación para convivencias saludables en contextos de vulnerabilidad escolar.
\end{abstract}

${ }^{\mathrm{a} C o r r e s p o n d e n c i a ~ a l ~ a u t o r ~}$

E-mail: jhonholguinalvarez@gmail.com 
Palabras clave: Artivismo popular; aprendizaje cooperativo; convivencia democrática; dáctilo pintura; teatro educativo.

\begin{abstract}
The objective of this study is to verify the effects of artivist pedagogies on democratic coexistence and cooperative learning in schoolchildren in vulnerable contexts. The approach was quantitative, experimental design. Two experiments were structured with experimental samples of: a) 85 students from fourth to sixth grade of Primary Education, b) 65 from sixth grade of Primary Education. It was found that: 1) popular artivist pedagogy based on cultural aspects (problems and folklore) develops democratic coexistence in vulnerable contexts, 2) pedagogy based on dactyl theatrical painting develops cooperative learning in a meaningful way and its dimensions, although the interaction Individual is a complex aspect to develop. Therefore, it was determined that popular artivist pedagogy and the experience of dactyl theatrical painting are useful pedagogies for the development of democracy and cooperation for healthy living in contexts of school vulnerability.
\end{abstract}

Keywords: Popular artivism; cooperative learning; democratic coexistence; dactyl paint; educational theater.

\title{
Introducción
}

La escuela tiene por visión construir las bases de la sociedad, en la cual el ser humano se constituye como parte de su estructura. En esta transición, la escuela depende de una formación escolar estable, con la cual desarrollar las dimensiones valorativa, emocional y cognitiva. En este sentido, los procesos escolares buscan la formación integral humana de forma constante mediante el desarrollo de una educación que aporte en la satisfacción de las necesidades humanas, en la generación de convivencias positivas, como también provocar aprendizajes cooperativos saludables. Muchas veces, esto resulta ser un supuesto en vez de una realidad, sobre todo si se diferencian las características coyunturales de contextos influenciados por otras variables intervinientes como: nivel social, nivel económico, comportamiento social y marco axiológico humano.

Uno de los problemas asociados a los de aprendizaje en convivencias y aprendizajes cooperativos, se circunscriben en la democracia, conflictividad y agresividad. Al respecto, estudios trasnacionales y nacionales han representado al estudiante peruano como un ser incapaz de decidir por su cuenta, o, en todo caso, en un sujeto con capacidades para decidir por condicionantes externos (compañeros más cercanos). Más del $40 \%$ de alumnos en el Perú siguen esta última consigna, y casi el $20 \%$ del total son poco participativos en 
competencias ciudadanas (Proyecto Educativo Metropolitano, PEM, 2014). Otra de las problemáticas son la agresividad y el conflicto tanto en el contexto familiar como en el social, estas influyen en gran medida en comunidades escolares en distintas modalidades. Un ejemplo son los más de 3000 casos de agresividad docente - alumno, directivo - alumno, alumno - alumno, que se han suscitado hasta antes del año 2017. No obstante, se visualizaron más de 2000 casos de conflictividad en los niveles de inicial, primaria y secundaria de educación básica regular al año 2018 (Ministerio de Educación del Perú, Minedu, 2018). Este problema ha acaecido en el $47 \%$ de miembros de instituciones educativas (directivos, coordinadores, profesores) a escolares peruanos: el $53 \%$ ocurre entre escolares; de esto, el 8 $\%$ son sujetos de educación inicial, el $36 \%$ del nivel primaria, y el $55 \%$ en educación secundaria (Minedu, 2019). Cabe señalar que más de 20000 casos ocurren en alguna interacción física, y se reproduce en los estudiantes en la violencia física, y más de 10000 casos en violencia psicológica (Minedu, 2019).

Estos problemas aparecen en distintos contextos de vulnerabilidad, en particular, en aquellos en que los indicadores más comunes son la delincuencia, pandillaje, robos, violencia social y rechazo cultural. Ante esta descripción coyuntural, los contextos vulnerables representan oportunidades para desarrollar distintos aspectos humanos: proactividad, resiliencia, asertividad, superación, emocionalidad; entre otros. La convivencia y el aprendizaje cooperativo en estos contextos representan oportunidades para probar pedagogías actuales de modos más fehacientes en la investigación, en situaciones de aprendizaje más reales o más palpables. Por cuanto, las estrategias de los docentes por lo general, son muy generalizables, buscan atender bajo metodologías que abarquen a todos los estudiantes, dejando de lado la atención personal que deben aplicar, más aún en grupos sociales que implican alguna emergencia social (Chib, Bentley y Wardoyo, 2019), generándose exclusión social, digital y hasta espiritual.

Las condiciones sociales, familiares y comunitarias permitirían establecer temas transversales en currículos de enseñanza más activa en estos contextos para abordar las problemáticas del alumnado (Carrillo et al., 2018), que aún no se solucionan a nivel del estado, si se tiene en cuenta que el enfoque en convivencias y cooperación son importantes en medios con coyunturas orientadas al desarrollo humano, por lo cual nacieron las siguie ntes interrogantes en el estudio: ¿Existe la posibilidad de estructurar estrategias contextualizadas 
para sociedades en emergencia social? ¿Qué tan efectivas pueden ser las estrategias activas y representativas en escolares que conviven en contextos vulnerables? ¿Llevarlos a ejercer estrategias de sociales por su cuenta, les permitirá desarrollar aprendizajes cooperativos y convivencias pacificas en sus propias sociedades de origen? ¿Cuáles serían los beneficios en su desarrollo escolar si aplican métodos basados en la crítica de sus sociedades?

\section{El artivismo: conceptos y fundamentos}

El artivismo es una actividad social y crítica convergente con el arte y el activis mo (Aladro-Vico, Jivkova-Semova y Bailey, 2018; Mesías-Lerma, 2018; Jivkova, Aladro y Sosa, 2019). También es considerada como la actividad multidisciplinaria, que implica el uso de distintas disciplinas (teatro, música urbana, oratoria, iconografía, grafitis, expresionis mo, entre otros) las cuales emergen en el contexto europeo (Aladro-Vico, Jivkova-Semova y Bailey, 2018; Mesías-Lerma, 2018), para hacer su aparición en el contexto latino, prescribiéndose como estímulos de la expresión urbana y la expresión del pensamiento crítico en comunidades vulnerables. Este arte involucra ideas del activismo como medio de transposición de ideas en integrantes de comunidades que concuerdan respecto a alguna situación social, con el fin de establecer medios de reacción, medios de transformación o medios de cuestionamiento social. Estos medios son las fuentes por las que el ser humano crea una cuestión crítica desde el contexto para el contexto en vulnerabilidad. El artivis mo utiliza el lenguaje estilístico expresionista para transmitir ideas, representarlas desde la problemática del contexto (figura 1), en el cual la urgencia social es de alta importancia para los pobladores como para los artivistas. En este sentido, el artivismo incluye contenidos de influencia crítico social (Aladro-Vico, Jivkova-Semova y Bailey, 2018; Jivkova, Aladro y Sosa, 2019), como también implica el uso de los significados del problema social para elaborar alguna forma de abordaje desde la propia esencia o base del problema (Aladro-Vico; Jivkova-Semova y Bailey, 2018; Macaya y Valero, 2019; Mesías-Lerma, 2018) y buscar la transformación de la realidad social mediante su abordaje socio crítico incluyendo distintos significados culturales (figura 1). 


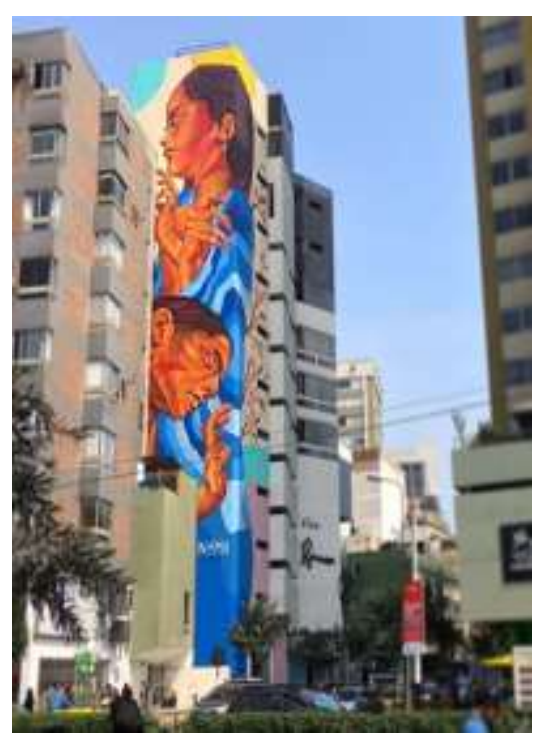

A*

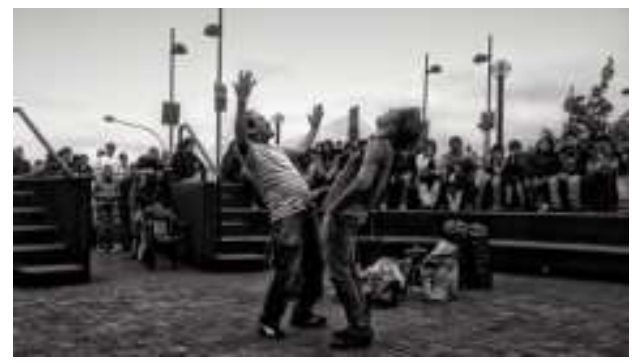

$\mathrm{B}^{*}$

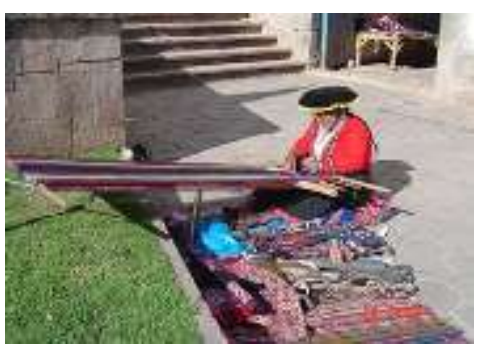

$\mathrm{C}^{*}$

Figura 1. Expresiones de artivismo mediante contenidos de la cultura peruana, arte, popular y expresivo del pensamiento social.

Fuente: $\quad \mathrm{A} *$ (https://gestion.pe/especial/zonalounge/arte-y-diseno/fotos-arte-urbano-lima-conozca-sus-obrasemblematicas-noticia-1994643); $\quad$ B* (https://laberintosuburbanos.wordpress.com/tag/comicos -ambulanteslmenos/); C*( https://pablogui.blogs pot.com/2017/04/el-arte-popular-peruano.html).

Nota: A*: Alma, trabajo de Pésimo Dmjc en Miraflores, Lima; B*: Cómicos ambulantes en plaza Chabuca Granda de Lima; $C^{*}$ : Textilería Ayacuchana, representación del arte andino popular en la capital de Perú.

El artivismo tiene sus fundamentos en el activismo (figura 2), el cual mediatiza la aparición de nuevas formas de expresión artística ante diversas injusticias políticas y sociales (Aladro-Vico; Jivkova-Semova y Bailey, 2018; Elliott, Silverman y Bowman, 2016). La confrontación social permite que los sujetos artivistas desarrollen nuevas formas de interacción con el público receptor con el fin de sumergir en ellos pensamientos, activarlos y provocar su accionar (Bradshaw, 2016; Bernárdez, Padilla y Sosa, 2019; Mesías-Lerma, 2018; Tello y Obando, 2019), así como ha ocurrido en contextos educativos en los cuales se desarrollaron nuevas formas de accionar ante alguna realidad coyuntural o tema transversal (Aran-Ramspott, Fedele y Tarrago, 2018; Bernárdez, Padilla y Sosa, 2019; Gass et al., 2016). 


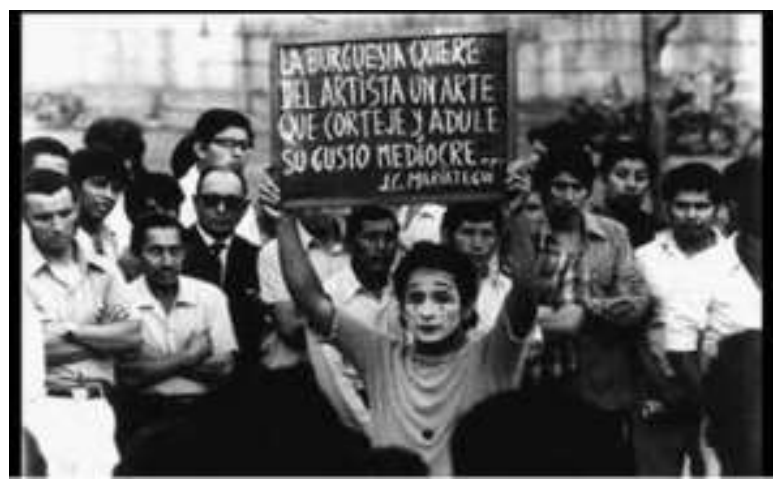

D*

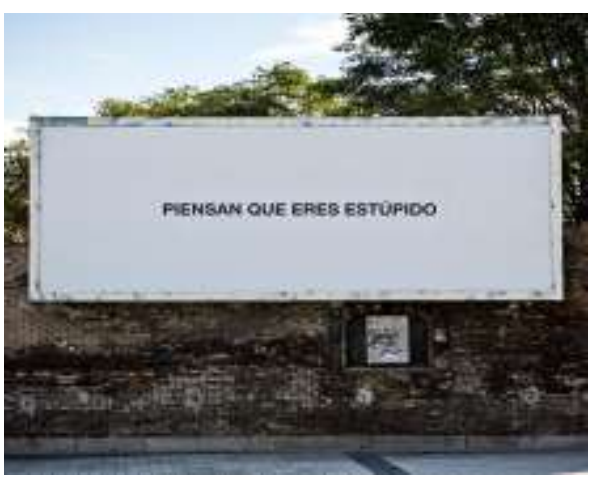

$\mathrm{E}^{*}$

Figura 2. Artivismo en la calle, formulados como representación activista en contra de la conyuntura social.

Fuente: D* (https://publimetro.pe/actualidad/que-les-gusta-tanto-peruanos-comicos-ambulantes-92792noticia/?ref=pur); E* (http://spy-urbanart.com/work/ellos/).

Nota: D*: Jorge Acuña, el fundador del espectáculo de comicidad ambulante (año 1963). E*: Ellos, anuncio en Zaragoza, España (año 2013).

En cuanto al artivismo popular desde la concepción socio educativa, se define como el acto de introducir los significados sociales (información social) para llevarlos a la sociedad, mediante fuentes basadas en disciplinas representativas que la propia sociedad utiliza para diferentes actividades (arte, teatro, música, entre otros), con fines de reeducarla y reconstruirla. La practicidad de este tipo de artivismo puede aparecer en las calles, debido a la falta de actos sociales que sean determinantes para el propio cambio social, expresionis mo de culturas excluidas, como medio de sustento laboral mediante el arte callejero, o, ante la falta de reacciones comunitarias ante injusticias políticas. La actividad artivista representa la oportunidad de hacer aparecer al hombre propiamente transfigurado en el sentido del ser humano como tal, valorativo en espíritu y pensador activo.

En relación a los efectos del artivismo popular en la sociedad para el cambio, se deben tener en cuenta cuatro principios correspondientes a distintos fenómenos que representan la construcción social en la interacción entre la escuela y la sociedad, por ello el artivismo: a) debe implicar su construcción por todos y para todos (fenómeno de arraigo social), b) el acto de artivismo debe ser digno de evaluarse así mismo para encontrar sus propias falencias (fenómeno de poder autocrítico), c) solo encuentra su sentido en la coyuntura social (fenómeno de inmersión social), y d) debe ser capaz de generar nuevas ideas en la población receptora con el fin de independizarse de sus creadores/artivistas (fenómeno de reproducción 
social). Las bases del artivismo provienen de las teorías de aprendizaje socio cultural y reproducción social (Bandura, 1979; Vigotsky, 1978), de las necesidades humanas (Maslow, 1991). Todas provienen de las bases fenomenológicas de la construcción social que remiten al significado humano como una forma de construcción de la sociedad como su significado simbólico (Schütz en Mélich, 1996, p. 37), y a su vez, el ser humano es parte de la construcción de la sociedad como parte de su estructura real o de su realidad (Berger y Luckmann en Niemann, 2005). Ninguno de estos componentes sociales puede existir sin el otro (humano y estructura social), es por esto que el artivismo funciona como medio de reconstrucción más allá del sentido de construcción social.

\section{Evidencias en artivismo popular y la dáctilo-pintura-teatral (DPT)}

Las evidencias empíricas en cuanto al artivismo desde el enfoque socio educativo, remiten resultados en los que los estudiantes lograron desarrollar actitudes y competencias de auto formación social y en las cuales buscaron desarrollar el sentido de auto reconstrucción mediante la aplicación de disciplinas del contexto peruano que integren el arte urbano, teatro, o la música (Grau y García-Raga, 2017a; Mesías-Lema, 2018; Tello y Obando, 2019). En otros casos, se han encontrado algunos aportes relacionadas al artivis mo popular, mediante el desarrollo de la cooperación para la mejora de convivencias saludables y promoción de la interacción escolar (Aladro-Vico, Jivkova-Semova y Bailey, 2018; Grau y García-Raga, 2017b; Grau, García-Raga y López-Martín, 2016). Las investigaciones que trabajaron el uso de contenidos sociales mediante la transmisión contenidos en medios sociales y de interacción virtual (Altun, 2015; Aran-Ramspott, Fedele y Tarrago, 2018; Huang et al., 2017), hallaron mejor impacto de cambio en sus receptores mediante estrategias de virtualización (Aran-Ramspott, Fedele y Tarrago, 2018; Uriz, y Esteve-Mon, 2016). Sin embargo, aún no se ha profundizado en alguna forma de artivismo popular que introduzca características sociales populares en contextos vulnerables con diferentes rasgos de obstrucción del desarrollo de la paz social.

Por otro lado, los hallazgos relacionados a la dáctilo-pintura-teatral (DPT en adelante), datan de los encontrados en Asia por Kim (2015), quien trabajó la educación basada en el arte para el desarrollo de las expresiones culturales y el fomento de etapas resilientes en el crecimiento estudiantil, sus evidencias declaran que la educación artística con múltiples 
formas de expresividad aminora el conflicto entre pares, con sostenibilidad en cuanto a otras variables como el optimismo y la creatividad. Estos resultados también se configuraron en otras evidencias en las cuales se incluyeron estudiantes con dificultades físicas y lograron fortalecer su sentido participativo al superarlas mediante experiencias personales y grupales (Greene, 2018; Samah, 2016), y otros hallazgos reportan mejor aceptabilidad de los problemas sociales, su tolerancia; y restitución del sentido comportamental positivo, mediante la vivencia de escenarios teatrales con significados contextuales o la inmersión en actividades artístico plásticas (García, Parada y Ossa, 2017; Greene, 2018; Van, Quintero y Pizzutto, 2018); fortaleciendo su expresividad en el uso de la pintura, elementos comunicativos también representados en el canto colectivo (Gelabert, 2017; Van, Quintero y Pizzutto, 2018), aunque no existen diferencias con los efectos formativos de otros estudios actuales que implicaron el uso de la pintura digital (Loughlin, 2015; Samah, 2016), la cual permite mejor sentido de la interpretación, creatividad; inclusive, en la comprensión textual.

La DTP es importante para la etapa escolar debido a que parte del juego como elemento básico para el aprendizaje y el desarrollo de nuevas competencias basadas en Groos (2012) y Uberman (1998). El juego se convierte en el nexo de viabilidad entre el profesorado y el alumnado para entender significados sociales, justamente en personas que se ubican en contextos que impiden tener relaciones sociales sanas, como lo puede ser la vulnerabilidad social o económica. Bajo estos preceptos, se logran en el niño otras influencias que bloquean distintos factores que trae consigo (eventos familiares negativos, problemas con amistades, autoritarismo en el aula, agresividad, alimentación, entre otros), por cuanto, el juego como base de la DPT, estimula en el desarrollo de: a) actitudes positivas, b) concentración, y, c) creatividad y pensamiento. La DPT es una actividad que recoge el significado social para transferirlo en contenidos representacionales a nivel artístico al público receptor (el que necesita cambiar) o en el mismo representante (el que se está formando).

El sentido de formación por actividades lúdicas permite considerar a la DTP como el medio creativo, corporal y espiritual que viabiliza la expresión de emociones y conocimie ntos mediante modelos mentales construidos de los significados pre existentes en la memoria sobre los significados más comunes evidenciados en el contexto en que el ser humano convive con otros, esta es una forma de potenciar las habilidades artísticas (Andrade y Durán, 2017; Arguello, 2017; Ruiz, 2014), aunque tiene sus bases en Shaw (1996), quien enfatizó 
que la pintura debía representarse por medios corporales, y de cuyos recursos se represente la realidad, se utilice la totalidad del cuerpo, e intervengan diversas fuentes sensoriales (brazos, piel, sentido perceptivo), como también condiciones espirituales y actitudinales en el aprendizaje cooperativo. Ambas experiencias parten de bases pedagógicas con sentido humanista en búsqueda del pensamiento transformador social desde la escuela (Maslow, 1943; Rogers, 1995), las cuales se han introducido en el currículo escolar peruano durante casi 10 años con resultados beneficiosos, pero con ciertas debilidades en la construcción de convivencia en estados de vulnerabilidad, las cuales aún no se reflejan en resultados científicos actuales provenientes de dichos contextos.

\section{Convivencias democráticas y aprendizajes cooperativos}

La convivencia democrática escolar es el concepto construido desde la interacción social que realizan los sujetos integrantes de cualquier grupo estudiantil y se estructura en convivencias inclusivas y convivencia para la paz (Fierro et al., 2010 en Valdés, López y Chaparro, 2018). La interacción desarrollada en el aula permite a sus integrantes relacionarse por actividades democráticas, inclusivas y bajo tolerancia para convivir (Colcord, Mathur y Zucker, 2016; Redford, Desrochers y Hoyer, 2017); y así construir el sentido de comprensión mutua, cooperación, participación en el modelo mental sobre convivencia en el grupo estudiantil, con la finalidad de establecer formas de vida más coherentes con el derecho de los demás y de los propios deberes (Grau y Garcia-Raga, 2017a). Estas se esquematizan bajo prácticas que conllevan a la democracia en convivencia por el hecho de practicarla, aminorando las propias coyunturas sociales que los estudiantes traen a la escuela desde su relación con el entorno (Grau y Garcia-Raga, 2017a; 2017b; Valdés, López y Chaparro, 2018). En su sentido práctico, la convivencia democrática solo se aprende si se experime nta, monitorea y se generan andamiajes participativos.

Algunos estudios encontraron que las medidas para la prevención de influencias estructurales en la convivencia son aquellas que aportan desde el recojo de problemáticas basadas en conflictos, identificados en el entorno social estudiantil. Por otra parte, la formación en democracia y en normativas, también son componentes importantes en contextos de vulnerabilidad ante la hostilidad social, delincuencia u otras variables que afecten las competencias para vivir en el alumnado (Tirado y Conde, 2016), los cuales 
concuerdan con otros que presentaron variables similares al de contextos hostiles en los que las interrelaciones se fracturan debido a variables sujetas a otras que son factores externos a la escuela como: faltas disciplinarias, disrupción hacia la normativa, agresividad en la coexistencia pacífica (Morales y López, 2018).

Los aprendizajes cooperativos son aquellos que se originan en los andamiajes provocados entre los alumnos con desarrollo próximo y los estudiantes con desarrollo potencial en razón de las metas sociales como punto de adaptación al grupo humano y la adopción de conductas en interacción social. Estos tipos de aprendizajes se basan en la teoría sociocultural y del aprendizaje social (Bandura, 1979; Vygotski, 1978), como los procesos que permiten el desenvolvimiento estudiantil para los aprendizajes activos. Esta variable se relaciona a la convivencia democrática como el medio participativo en que se producen actividades como la participación y el ejercicio ético para el aprendizaje. En este enfoque, los aprendizajes cooperativos se conceptualizan como la ejecución de habilidades para el aprendizaje en convivencias saludables, regulado por interacciones mediante la búsqueda de conocimientos dominantes / establecidos y conocimientos potenciales / previos (Chikh y Hank, 2016; Mayordomo y Onrubia, 2015; Prova, 2017).

En este sentido, los aprendizajes cooperativos también implican componentes afectivos y motivacionales que medían y viabilizan las convivencias para el aprendizaje. En esta movilización de actividades, se ejercen competencias paralelas que comprometen a los estudiantes en la realización de otras, las cuales permiten fortalecen dicha viabilidad, cuyos aspectos son el cumplimiento sano de metas, adaptación al medio, compromiso de ayuda mutua, e interdependencia positiva (Mayordomo y Onrubia, 2015). Estas actividades se formulan como dimensiones del aprendizaje cooperativo. Sin embargo, otros autores la estructuraron por responsabilidad individual, habilidades sociales, evaluación de grupos (Johnson y Johnson en Prova, 2017), aunque las concepciones convergen en la capacidad de interdependencia positiva como un aspecto crucial para la ejecución de aprendizaje cooperativos (Chikh y Hank, 2016; Johnson y Johnson en Prova, 2017; Mayordomo y Onrubia, 2015), por cuanto se convierte en la dimensión eje de los aprendizajes cooperativos.

El objetivo de la investigación fue comprobar los efectos de pedagogías artivistas en la convivencia democrática y los aprendizajes cooperativos en contextos escolares en 
vulnerabilidad. Lo cual desencadenó las siguientes hipótesis del estudio: a) La pedagogía artivista popular desarrolla efectos de mejora en la convivencia democrática en escolares de educación primaria que provienen de contextos vulnerables; y b) La DTP como medio pedagógico de artivismo incrementa el aprendizaje cooperativo de estudiantes de primaria de contextos vulnerables de Lima.

\section{Metodología}

\section{Diseño de investigación}

La investigación se sitúa en el enfoque cuantitativo de diseño experimental, de nivel explicativo, debido a que se plantearon manipulaciones de la variable independiente para verificar sus efectos sobre la variable dependiente (Baena, 2014; Hernández et al., 2018). En este caso, se desarrollaron dos estudios experimentales (con grupo control (x) y grupo experimental (y)). En cuanto al primer estudio (Experimento A): se desarrolló la aplicación de la variable artivismo popular sobre la variable dependiente convivencia democrática. En el segundo estudio (Experimento B): se aplicó la variable independiente DTP sobre la variable aprendizaje cooperativo. Ambos grupos presentaron el planteamiento de medición pretest y postest para su comparación experimental.

\section{Experimento A}

Artivismo popular y convivencia democrática

\section{Sujetos}

La muestra probabilística se conformó por 85 estudiantes de primaria, de los grados cuarto $(M=9.5$ años de edad; D.E.= 0.3 años $)$, quinto $(M=10.32$; D.E. $=0.1)$ y sexto grado de primaria $(\mathrm{M}=11.2$; D.E. $=0.2)$. Todos provinieron de tres instituciones públicas ubicadas en tres contextos vulnerables del distrito de Comas (ciudad: Lima). Los criterios para la selección de estos contextos, permitieron seleccionar las tres escuelas participantes para la conformación de la población del estudio; y así lograr el ajuste a una distribución más equitativa respecto a cada contexto (tabla 1).

\section{Tabla 1.}

Criterios de selección y distribución de estudiantes de acuerdo a los contextos vulnerables de Comas, Lima 


\begin{tabular}{|c|c|c|c|c|}
\hline $\mathrm{N}^{\circ}$. & Contexto* & $\begin{array}{c}\text { Descripción socio } \\
\text { económica del contexto }\end{array}$ & $\begin{array}{l}\text { Grados en } \\
\text { Primaria }\end{array}$ & $\begin{array}{l}\text { Cantidad de } \\
\text { extracción }\end{array}$ \\
\hline 1 & $\begin{array}{l}\text { Asociación de } \\
\text { Vivienda } 1\end{array}$ & $\begin{array}{l}\text { Violencia juvenil, bajos } \\
\text { recursos económicos: } \\
\text { robos }\end{array}$ & $\begin{array}{l}\text { Cuarto } \\
\text { Quinto }\end{array}$ & 22 \\
\hline 2 & $\begin{array}{l}\text { Asociación de } \\
\text { Vivienda } 2\end{array}$ & $\begin{array}{l}\text { Violencia familiar, } \\
\text { pandillaje juvenil }\end{array}$ & Sexto & 20 \\
\hline 3 & $\begin{array}{l}\text { Asentamiento } \\
\text { Humano } 1\end{array}$ & $\begin{array}{l}\text { Escasos recursos } \\
\text { económicos; robos }\end{array}$ & Sexto & 22 \\
\hline 4 & $\begin{array}{l}\text { Asentamiento } \\
\text { Humano } 2\end{array}$ & $\begin{array}{l}\text { Escasos recursos } \\
\text { económicos; } \\
\text { delincuencia juvenil }\end{array}$ & $\begin{array}{l}\text { Cuarto } \\
\text { Sexto }\end{array}$ & 21 \\
\hline
\end{tabular}

Fuente: Caracterización basada en el registro de dirección de escuelas (fichas de inicio de matrícula en instituciones educativas).

Los sujetos se agruparon conforme a criterios metodológicos para conformar el grupo control $\left(\mathrm{n}_{(\mathrm{x})}=45\right)$ y el grupo experimental $(\mathrm{n}(\mathrm{y})=40)$. En cuanto a la distribución por género, la cantidad mayor fue del género masculino (m: $75 \%$; f: $25 \%$ ). Cabe señalar que todos los participantes fueron incluidos en el estudio mediante la firma de consentimiento informado otorgados por sus padres o por sus tutores de familia.

\section{Instrumento y procedimiento del experimento $\mathrm{A}$}

Para este estudio se utilizó la Escala de medición de la convivencia democrática ESMCD (Alcántara y Holguin, 2019), en su versión preliminar con el fin de establecer sus resultados en coherencia con las características de los sujetos del estudio, y así considerar su adecuación lingüística, estructuración metodológica; y pertinencia a la edad de los sujetos implicados en los grados de atención en el nivel primaria. El instrumento es de consistencia escalar, y estructura de cuestionario. Este se desarrolló hasta en 30 minutos como máximo. El instrumento se compone por 25 preguntas acompañadas por una escala de que tienen una escala de tipo politómica con puntuaciones ascendentes (del 1 al 3): Muy de acuerdo, de acuerdo, en desacuerdo. El instrumento está acondicionado para medir los componentes: a) colaboración y tolerancia, b) normativas para la convivencia, y c) resolución de conflictos.

\section{Tabla 2.}

Índices de confiabilidad Alfa de Cronbach y correlaciones 


\begin{tabular}{lcc}
\hline Componentes* & $\begin{array}{c}\text { Alfa de } \\
\text { Cronbach }\end{array}$ & $\begin{array}{c}\text { Convivencia } \\
\text { democrática (r) }\end{array}$ \\
\hline Colaboración y tolerancia &, 821 &, $845^{* *}$ \\
Normativas para la convivencia &, 789 &, $816^{* *}$ \\
Resolución de conflictos &, 895 &, $889 * *$ \\
\hline \multicolumn{2}{c}{ Fuente: Base de datos de la investigación.* (ESMC $=, 855) ; * *(p<.001)}$.
\end{tabular}

El análisis de confiabilidad se realizó a través de la aplicación piloto a 30 estudiantes, seleccionados de dos de los contextos vulnerables que conformaron parte del estudio en general (tabla 1). En razón del análisis de datos, sus resultados se consideraron aceptables para la medición cuantitativa. De igual modo, el análisis de correlaciones permitió aseverar su significancia estadística, imitando la forma correlación ítem - test a la forma correlación sub test - test (tabla 2). En cuanto a este análisis, se obtuvieron resultados coherentes en relación a los índices Alfa de Cronbach obtenidos.

El procedimiento del estudio se inició mediante la gestión de los proyectos de investigación estudiantil universitaria (etapa: fin de carrera) basadas en artivismo, desde las áreas de dirección nacional y de investigación de una Escuela Profesional de Educación Primaria de una universidad privada de Lima. Se logró plantear el proyecto de artivis mo popular y realizar el contacto y coordinaciones de visita pedagógica experimental con los directivos de las instituciones educativas. Se gestionó la aplicación de un plan de intervención experimental de 50 actividades pedagógicas de acuerdo a su naturaleza (actividades artivistas populares: 25; actividades de cuestionamiento social: 25) (figura 1). Este proyecto se aplicó durante un semestre académico y constó de la participación de los agentes de la escuela (alumnos, padres, docentes) en tres momentos: a) organización temática; b) generación de ideas artivistas; c) artivismo popular y callejero. 


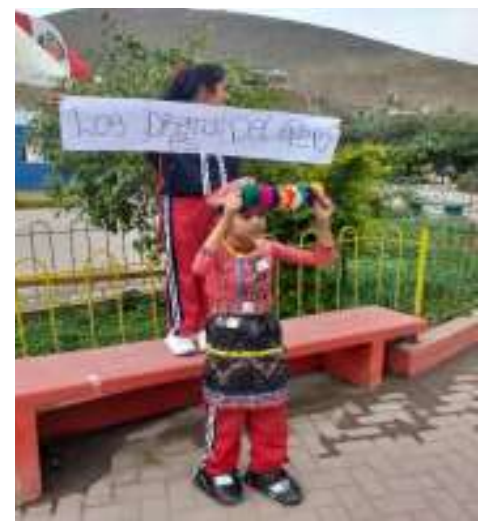

$\mathrm{A}^{*}$

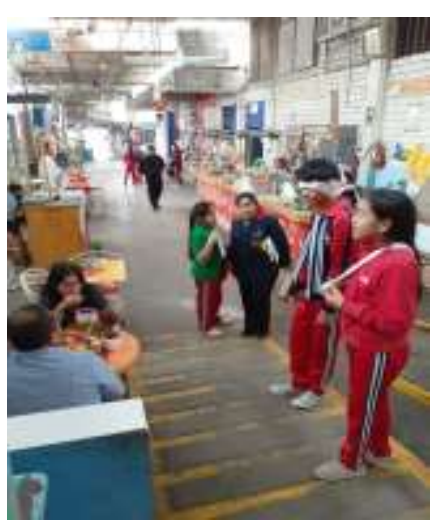

$\mathrm{B} *$

Actividad de cuestionamiento social

Figura 3. Ejemplificaciones del programa de artivismo popular.

Fuente: Elaboración propia.

Nota: A* (actividad artivista Diseñadores Perú); B* (Genoma xx).

El programa de artivismo popular se aplicó después de la distribución del cuestionario ESMCD a los estudiantes de cada grupo conformado. Para el trabajo con el grupo experimental, se organizó el desarrollo de este programa en cada sección respectiva a la escuela con los participantes de dicho grupo. La aplicación de cada etapa o fase pedagógica de artivismo popular se desarrolló durante 25 o 30 minutos, a excepción de la fase de artivismo popular y callejero, ya que esta duró hasta 3 horas, debido a las salidas de las instituciones educativas participantes. En cuanto a la organización temática, esta fase permitió el desarrollo de la organización de los estudiantes en el aula, en dos sub grupos: preparadores y aplicadores; en la fase generación de ideas artivistas, los sujetos determinaban las temáticas relacionadas a las problemáticas sociales y la modalidad de abarcar el problema en la fase final (teatro, representaciones gráficas, canto, bailes u otras que se ejecutarín de forma múltiple). Luego de cinco meses y medio, se aplicaron las respectivas pruebas de salida al grupo control y al grupo experimental. 


\section{Resultados}

\section{Artivismo popular y convivencia democrática}

\section{Tabla 3.}

Mediciones pretest y postest en t-Student de la convivencia democrática luego del programa de artivismo popular

\begin{tabular}{cccccc}
\hline Momentos & Grupos & $\mathrm{N}$ & Media & $\begin{array}{c}\text { Desv. } \\
\text { Desviación }\end{array}$ & $\begin{array}{c}\text { Desv. Error } \\
\text { promedio }\end{array}$ \\
\hline \multirow{2}{*}{ Pretest } & Control & 31 & 29.16 & 3.099 & 0.557 \\
& Experimental & 34 & 30.41 & 3.135 & 0.538 \\
\hline \multirow{2}{*}{ Postest } & Control & 31 & 31.65 & 3.136 & 0.563 \\
& Experimental & 34 & 64.41 & 6.679 & 1.145 \\
\hline
\end{tabular}

Fuente: Base de datos de la investigación.

La comparación de datos pretest entre los grupos experimental y control, reportó diferencias no significativas (tabla 3 , dif. $(x-y)=-1.250$; sig. $=0.11 ; p>.001)$. En cambio, luego de la aplicación del programa de artivismo popular, se obtuvieron promedios significativos en cuanto a los puntajes de la medición postest, los cuales fueron favorables al grupo experimental $(\mathrm{M}=64.41$; D.E. $=6.679)$ y en menor gado para los estudiantes del grupo control $(\mathrm{M}=31.65$; D.E.= 3.14). A su vez, los datos de esta comparación (postest) permitieron corroborar diferencias significativas entre ambos grupos $(\mathrm{t}(63)=-24.921$; sig . $=.000 ; p<.001)$.

\section{Aspectos dimensionales de la convivencia democrática}

\section{Tabla 4.}

Mediciones pretest y postest en t-Student de aspectos dimensionales de la convivencia democrática luego del programa de artivismo popular

\begin{tabular}{ccccccc}
\hline Dimensiones & Momentos & Grupos & $\mathrm{N}$ & Media & $\begin{array}{c}\text { Desv. } \\
\text { Desviación }\end{array}$ & $\begin{array}{c}\text { Desv. Error } \\
\text { promedio }\end{array}$ \\
\hline $\begin{array}{c}\text { Colaboración } \\
\text { y tolerancia }\end{array}$ & Pretest* & Control & 31 & 9.00 & 1.366 & 0.245 \\
& & Experimental & 34 & 9.76 & 1.478 & 0.254
\end{tabular}




\begin{tabular}{ccccccc} 
& Postest** & Control & 31 & 10.65 & 0.839 & 0.151 \\
& Pretest* & Control & 31 & 9.52 & 2.942 & 0.528 \\
\hline $\begin{array}{c}\text { Normativas } \\
\text { para la } \\
\text { convivencia }\end{array}$ & Postest** & Control & 31 & 10.23 & 1.820 & 0.327 \\
& Experimental & 34 & 22.53 & 3.287 & 0.564 \\
\hline $\begin{array}{c}\text { Resolución } \\
\text { de conflictos }\end{array}$ & Pretest* & Control & 31 & 12.16 & 1.614 & 0.290 \\
& Postest** & Experimental & 34 & 28.00 & 3.275 & 0.562 \\
& & Control & 31 & 9.26 & 1.825 & 0.328 \\
\hline & & Experimental & 34 & 13.88 & 2.345 & 0.402 \\
\hline
\end{tabular}

Fuente: Base de datos de la investigación.

Nota: $*(p>.001) ; * *(p<.001)$.

\section{Experimento B:}

DTP y aprendizaje cooperativo

\section{Sujetos}

La muestra se caracterizó por 65 estudiantes del sexto y quinto grado de primaria (M = 11.3 años de edad; D.E.= 0.2 años). Los estudiantes asistían a escuelas públicas de la provincia del Callao, residentes en el distrito de Ventanilla. Estos eran estudiantes de dos instituciones ubicadas en dos contextos vulnerables del distrito, ello con el fin de equiparar condiciones poblaciones con los sujetos del primer experimento. Estos estudiantes fueron seleccionados acorde a la caracterización realizada por los lugares en que habitaban (tabla $5)$. 
Tabla 5.

Criterios de selección y distribución de estudiantes de acuerdo a los contextos vulnerables en Ventanilla, Callao

\begin{tabular}{|c|c|c|c|c|}
\hline $\mathrm{N}^{\mathrm{o}}$. & Contexto* & $\begin{array}{l}\text { Descripción socio } \\
\text { económica del contexto }\end{array}$ & $\begin{array}{l}\text { Grados en } \\
\text { Primaria }\end{array}$ & $\begin{array}{c}\text { Cantidad de } \\
\text { extracción }\end{array}$ \\
\hline 1 & $\begin{array}{l}\text { Asociación de } \\
\text { Vivienda }\end{array}$ & $\begin{array}{lr}\text { Delincuencia } & \mathrm{y} \\
\text { pandillaje juvenil } & - \text { nivel } \\
\text { socioeconómico } & \text { muy } \\
\text { bajo } & \end{array}$ & $\begin{array}{l}\text { Quinto } \\
\text { Sexto }\end{array}$ & 34 \\
\hline 2 & $\begin{array}{l}\text { Asentamiento } \\
\text { Humano }\end{array}$ & $\begin{array}{l}\text { Delincuencia juvenil y } \\
\text { nivel socioeconómico } \\
\text { muy bajo }\end{array}$ & $\begin{array}{l}\text { Quinto } \\
\text { Sexto }\end{array}$ & 31 \\
\hline
\end{tabular}

Fuente: Caracterización basada en el registro de matrículas de las instituciones educativas .

Los sujetos fueron asignados a cada submuestra, por lo cual, el grupo control (n (x)= 33) y el grupo experimental $(n(y)=32)$ alcanzaron medidas muy semejantes entre sí. En cuanto al género, el $45 \%$ perteneció al género masculino y $55 \%$ al género femenino. Todos los participantes lograron implicarse en el estudio mediante la aceptación o permiso de sus padres. A través de la gestión del docente de aula se hizo llegar los documentos del consentimiento informado con el fin que los padres devolviesen las fichas respectivas a los docentes de cada escuela antes de ejecutar el estudio.

\section{Instrumento y procedimiento del experimento B}

Para la medición de la variable, se aplicó el Cuestionario de evaluación del aprendizaje cooperativo - APRECOOP (ad hoc), constituido por 55 ítems con respuestas escalares a los que se asignan valores del 1 al 3: Nunca, siempre, a veces. El cuestionario es de aplicación colectiva y se realiza durante 15 minutos como máximo. Las dimensiones que abarca en cuanto a su contenido son: a) interacción positiva, b) responsabilidad individual, c) interacción auténtica cara a cara, d) habilidades para la socialización, e) evaluación individual y grupal. Antes del experimento, se aplicó una versión preliminar a 25 estudiantes del Callao, con similares condiciones al experimento anterior. Estos asistían a escuelas públicas pertenecientes a instituciones educativas públicas de la misma provincia. Los datos obtenidos permitieron obtener índices Alfa de Cronbach que permitían aducir que el instrumento a nivel de contenido de la variable y las dimensiones eran estables para la 
investigación (aprendizaje cooperativo = 850; interdependencia positiva = ,769; responsabilidad individual $=, 821$; habilidades para la socialización $=, 798$; evaluación individual y grupal $=, 835)$.

Una vez confirmada la validez y confiabilidad del instrumento y agrupados las aulas metodológicas (experimental y control), este se aplicó en los sujetos para evaluar su aprendizaje cooperativo en medición pretest, una vez gestionadas las visitas a las instituciones educativas de origen estudiantil. El programa DTP se implementó por 45 sesiones de aprendizaje con estructura de: inicio, proceso y salida, esto para hacerlas más coherentes con la programación habitual que los docentes desarrollaban en sus aulas, cuya exigencia era establecida por el Ministerio de Educación del Perú. Cada una de las sesiones también implicaba la ejecución de procesos metodológicos determinados por un tiempo (entre 45 y 50 minutos): a) Organización de temáticas de abordaje, b) Aplicación compartida, c) Representación gráfica y teatral. Las aplicaciones b y c se desarrollaron en modalidad personalista, por duetos o en grupos de cuatro o más personas escolares (figura 4). Aunque este programa también introdujo a los padres o tutores, se diferenció del anterior (artivismo popular) en especial a que su presencia se implicase en más del $80 \%$ de sesiones, con el fin de evitar influencias emocionales en lo que los sujetos deseaban expresar en sus representaciones gráficas como también en la ejecución de actividades teatrales (por ejemplo, para evitar el pánico escénico).

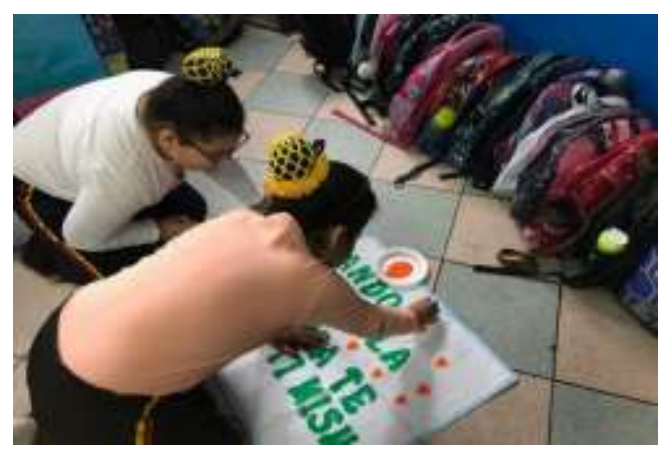

C*

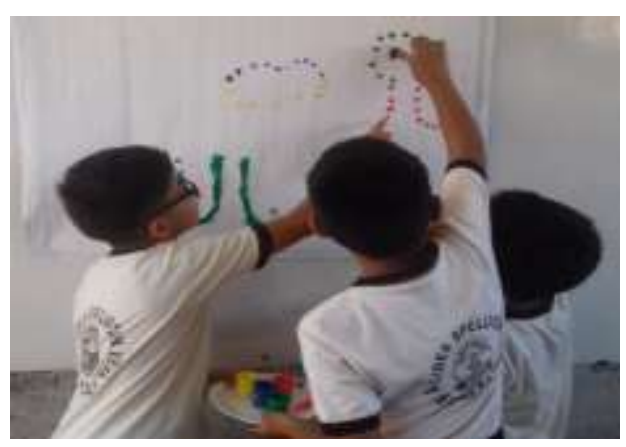

D* 


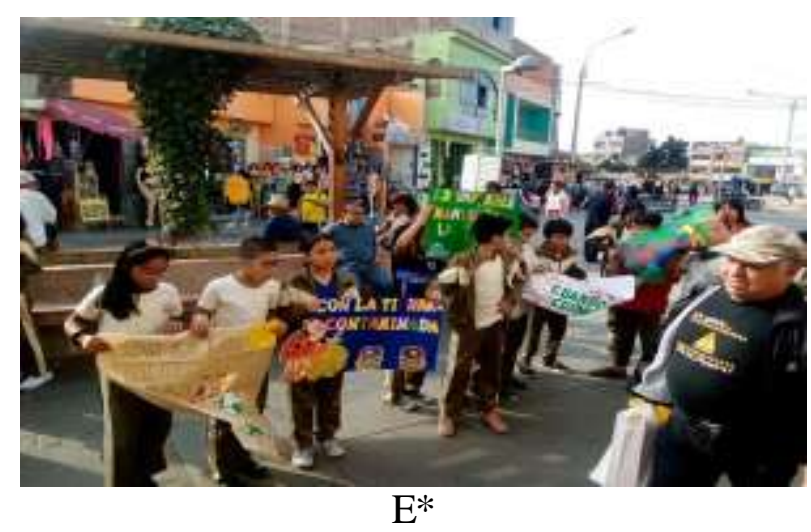

Figura 4. Actividades del programa de DTP en contextos de Ventanilla, Perú.

Fuente: Elaboración propia.

Nota: C* (Actividad Defiéndete tú misma); D* (Manos libres, camino libre); E* (Plazuela de Ventanilla: actividad Memoria de mujeres, memoria de hombres).

La aplicación del programa terminó por realizarse a mediados del mes de diciembre. El instrumento APRECOOP se aplicó por segunda vez (medición postest) al finalizar el programa, los datos obtenidos se analizaron mediante el programa estadístico KolmogorovSmirnov con el fin de determinar si estos demostraban distribución normal o presentaban ausencia de ella. Esto permitió decidir el programa estadístico más asequible para la comparación de grupos metodológicos.

\section{Resultados del Aprendizaje cooperativo}

\section{Tabla 6.}

Mediciones pretest y postest en $t$-Student de aprendizaje cooperativo luego dell programa DTP

\begin{tabular}{cccccc}
\hline Momentos & Grupos & $\mathrm{N}$ & Media & $\begin{array}{c}\text { Desv. } \\
\text { Desviación }\end{array}$ & $\begin{array}{c}\text { Desv. Error } \\
\text { promedio }\end{array}$ \\
\hline \multirow{2}{*}{ Pretest } & Control & 33 & 70.42 & 6.615 & 1.151 \\
& Experimental & 32 & 70.69 & 6.322 & 1.118 \\
\hline \multirow{2}{*}{ Postest } & Control & 33 & 79.97 & 6.177 & 1.075 \\
& Experimental & 32 & 131.72 & 8.781 & 1.552 \\
\hline
\end{tabular}

Fuente: Base de datos de la investigación. 
La comparación de los datos obtenidos antes de aplicar el programa DTP (medición pretest) (tabla 6), presentó diferencias leves entre los puntajes obtenidos en el grupo experimental y control (dif. $=-0,263$ ). Estas mediciones señalaron estabilidad al iniciar el experimento, y aplicar las actividades en el grupo experimental. Luego de la experimentación, la diferencia entre los puntajes de ambos grupos reportó la diferencia favorable al grupo que recibió las actividades de dáctilo pintura teatral (dif. $=-51.759$ ). Este reporte fue significativo en la prueba $\mathrm{t}-$ Student $(\mathrm{t}(63)=-27,549 ; \mathrm{sig}$. $=.000 ; p<.001)$. Por cuanto, existieron evidencias que permitieron aceptar que el programa de intervención pedagógica incrementó el aprendizaje cooperativo en los estudiantes residentes en Ventanilla.

\section{Aspectos dimensionales del aprendizaje cooperativo}

\section{Tabla 7.}

Mediciones pretest y postest en $t$-Student de aspectos dimensionales del aprendizaje cooperativo luego del programa DTP

\begin{tabular}{|c|c|c|c|c|c|c|}
\hline Dimensiones & Momentos & Grupos & $\mathrm{N}$ & Media & $\begin{array}{c}\text { Desv. } \\
\text { Desviación }\end{array}$ & $\begin{array}{c}\text { Desv. Error } \\
\text { promedio }\end{array}$ \\
\hline \multirow{4}{*}{$\begin{array}{l}\text { Interacción } \\
\text { positiva }\end{array}$} & \multirow[t]{2}{*}{ Pretest* } & Control & 33 & 9.12 & 1.364 & 0.237 \\
\hline & & Experimental & 32 & 9.19 & 1.330 & 0.235 \\
\hline & \multirow{2}{*}{ Postest* } & Control & 33 & 19.09 & 2.590 & 0.451 \\
\hline & & Experimental & 32 & 17.84 & 1.798 & 0.318 \\
\hline \multirow{4}{*}{$\begin{array}{c}\text { Interacción } \\
\text { auténtica cara a } \\
\text { cara }\end{array}$} & \multirow[t]{2}{*}{ Pretest* } & Control & 33 & 15.67 & 2.146 & 0.374 \\
\hline & & Experimental & 32 & 15.75 & 2.258 & 0.399 \\
\hline & \multirow{2}{*}{ Postest** } & Control & 33 & 16.82 & 2.214 & 0.385 \\
\hline & & Experimental & 32 & 17.00 & 1.984 & 0.351 \\
\hline \multirow{4}{*}{$\begin{array}{l}\text { Responsabilidad } \\
\text { individual }\end{array}$} & \multirow[t]{2}{*}{ Pretest* } & Control & 33 & 9.73 & 1.755 & 0.305 \\
\hline & & Experimental & 32 & 10.91 & 2.220 & 0.392 \\
\hline & \multirow{2}{*}{ Postest** } & Control & 33 & 9.12 & 1.364 & 0.237 \\
\hline & & Experimental & 32 & 9.19 & 1.330 & 0.235 \\
\hline
\end{tabular}




\begin{tabular}{ccccccc}
\hline & Pretest* & Control & 33 & 19.09 & 2.590 & 0.451 \\
$\begin{array}{c}\text { Habilidades para } \\
\text { la socialización }\end{array}$ & & Experimental & 32 & 17.84 & 1.798 & 0.318 \\
& Postest** & Control & 33 & 15.67 & 2.146 & 0.374 \\
& Experimental & 32 & 15.75 & 2.258 & 0.399 \\
\hline \multirow{2}{*}{$\begin{array}{c}\text { Evaluación } \\
\text { individual y } \\
\text { grupal }\end{array}$} & Pretest* & Control & 33 & 16.82 & 2.214 & 0.385 \\
& & Experimental & 32 & 17.00 & 1.984 & 0.351 \\
& Postest** & Control & 33 & 9.73 & 1.755 & 0.305 \\
\hline
\end{tabular}

Fuente: Base de datos de la investigación.

Nota: $*(p>.001) ; * *(p<.001)$.

\section{Discusión}

El objetivo del estudio fue comprobar los efectos de pedagogías artivistas para mejorar convivencias democráticas y aprendizajes cooperativos, mediante el planteamiento de dos hipótesis comprobadas mediante la realización de dos estudios empíricos. De acuerdo a la convivencia democrática, los resultados probados mediante estadística paramétrica, también fueron establecidos mediante estadística no paramétrica, obteniéndose resultados similares en cuanto a estabilidad entre grupos, como también al finalizar el experimento, con medianas favorables para el grupo de estudiantes que recibió el programa de artivis mo popular / grupo experimental $(M d n$ (pretest) $=29,00 ; M d n$ (postest) $=45,00)$; a su vez, estas diferencias intergrupales fueron significativas $(Z=-6,931 ; p<.001)$. Estos hallazgos han demostrado a nivel de convivencia democrática, que se ha desarrollado acorde a los planteamientos socio educativos que toda institución educativa trabaja mediante la visión y misión escolar (Bandura, 1979; Vigotsky, 1978), y como pensamiento pedagógico contemporáneo sobre la construcción de sociedades en búsqueda de la satisfacción de necesidades (Maslow, 1991), y la conservación de otras concepciones como, ciudadanía, sentido de sociedad y aculturación (Berger y Luckmann en Niemann, 2005; Schütz en Mélich, 1996).

Respecto a las evidencias experimentales, los resultados obtenidos en niños de contextos vulnerables de Comas, lograron desarrollar decisiones democráticas más ajustadas 
a las normativas del aula, como también, a la normativa escolar como en otros estudios en que se introdujo variables sociales en el ámbito escolar para practicarlas de forma vivencial (Colcord, Mathur y Zucker, 2016; Grau y García, 2017a; 2017b; Fedele y Tarrago, 2018; Huang et al., 2017; Redford, Desrochers y Hoyer, 2017). En la convivencia democrática ha ejercido mayor influencia el sentido cultural que el artivismo implicó en actividades de práctica social o las correspondientes al aprendizaje socio cultural. La práctica en las calles, llevar el sentido de la cosmovisión andina a las calles, la presencia de los signos o significados culturales de la selva o la costa, con contenidos coyunturales ha hecho efecto en los propios estudiantes en la forma de convivir desde antes de salir de la escuela, inclusive desde el hecho de programarse la construcción del mensaje activista, la construcción de materiales y la obtención de recursos de forma democrática; son evidencias obtenidas bajo estímulos originarios del artivismo europeo adaptado al contexto peruano.

La diferencia de medias también fue significativa en las puntuaciones obtenidas por cada dimensión de la convivencia escolar luego de aplicar el programa de artivismo popular: colaboración y tolerancia en la convivencia democrática escolar, (dif. (y) $=-12,304 ; \mathrm{t}(63)=$ $-18,418 ; p<.001$ ); normativa para la convivencia (dif. $(\mathrm{y})=-15.839 ; \mathrm{t}(63)=-24,351 ; p<.001$ ); resolución de conflictos (dif. $(y)=-4,624 ; \mathrm{t}(63)=-8,810 ; p<.001)$. Estas evidencias se basan en resultados de otros estudios que plantearon que el artivismo es una forma de expresión cultural que desarrollar distintas formas de expresividad en el aula y como formas de activismo determinadas por la problemática contextual (Aladro-Vico, Jivkova-Semova y Bailey, 2018; Aran-Ramspott, Fedele y Tarrago, 2018; Bradshaw, 2016; Bernárdez, Padilla y Sosa, 2019; Bernárdez, Padilla y Sosa, 2019; Gass et al., 2016). Esto ha evidenciado que los estudiantes desarrollaron formas de adecuación a la normativa de convivencia en sus respectivas escuelas (Grau y Garcia-Raga, 2017a;2017b), lo cual se ha establecido en actividades propias de colaboración e interacción entre estudiantes. Por otro lado, es importante notar que el lugar de procedencia permitió a los estudiantes menos democráticos desarrollar conductas de mayor adecuación a las conductas de otros más activos en el aula, quienes, por adecuarse rápidamente a las normativas que el profesor, establecen mejores hábitos para la aplicar actos democráticos que los demás. Entonces, esta experimentación mediante el artivismo los adecuó a ambientes de aprendizaje democrático que les permitieron 
elegir bajo procesos de tolerancia, y, resolver conflictos como lo evidencian las puntuaciones de esta parte del estudio.

Respecto a las evidencias del programa DTP como medio pedagógico experimental para desarrollar los aprendizajes cooperativos, se encontraron los resultados que fueron comprobados a nivel no estadístico no paramétrico. La diferencia de medianas en el grupo experimental fue evidente entre el momento inicial y final de aplicación del programa ( $M d n$ (pretest) $=71,00 ; M d n$ (postest) $=92,00)$, como también fueron significativas $(\mathrm{Z}=-6,931 ; p<.001)$. Estos resultados presentan características semejantes a otros estudios con métodos experimentales similares a la DTP, permitieron disminuir la conflictividad en el aula, acrecentar el optimis mo como también desarrollar comportamientos sociales más adaptados a la interacción entre estudiantes (Greene, 2018; Kim, 2015), representando en este estudio modelos de aprendizaje cooperativos (Gelabert, 2017; Loughlin, 2015; Van, Quintero y Pizzutto, 2018), eficientes, y con crecimiento significativo. Estas evidencias permitieron aseverar que como medio pedagógico artivista, la DTP permitió que los sujetos desarrollasen aprendizajes compartidos, reflexiones y conductuación positiva como se predijo desde los enfoques planteados por estudios originarios del artivismo (Bradshaw, 2016; Bernárdez, Padilla y Sosa, 2019; Gass et al., 2016; Mesías-Lerma, 2018; Tello y Obando, 2019).

Los resultados de la medición postest, respecto a las dimensiones del aprendizaje cooperativo, presentaron diferencias no paramétricas con medianas favorables al grupo experimental (responsabilidad individual $(M d n(\mathrm{y}))=26,00$; interacción positiva $(M d n(\mathrm{y}))=$ 24,00 ; habilidades sociales $(M d n(\mathrm{y}))=26,00$; evaluación individual y grupal $(M d n(\mathrm{y}))=$ 15,00). Todas estas diferencias fueron significativas (responsabilidad individual $(Z)=-6,951$; $p<.001$; interacción positiva $(Z)=-6,866 ; p<.001$; habilidades sociales $(Z)=-6,939 ; p<.001$; evaluación individual y grupal $(\mathrm{Z})=-6,715 ; p<.001)$. Estas evidencias permitieron aducir que el cambio se debió a la aplicación del programa DTP, y se extrapolan a estudios similares que declararon que la pintura y el medio corporal introduce nexos de cooperación e interrelaciones pacificas importantes entre compañeros de grupo que buscan dotar de significados a sus propias ideas (Andrade y Durán, 2017; Arguello, 2017; Morales y López, 2018), como también se fortalecen los conocimientos compartidos, tratando de ser menos dominantes (Chikh y Hank, 2016; Mayordomo y Onrubia, 2015; Prova, 2017), tal como ocurrió en esta experiencia de DTP, generándose en los estudiantes de este grupo más 
responsabilidades grupales, compromisos, poder para la autoevaluación, y la capacidad de autocrítica. Esto último se ha asemejado al criterio de existencia del artivismo entre la experiencia que vivieron los sujetos del estudio: su permanencia en la transformación social solo sobrevive si se autoevalúa en sociedad, ya que su fin único es construirla o reconstruirla.

En función a la dimensión interacción positiva, se calcularon índices respecto a su comparación en la medición de grupos, por cuanto tampoco se alcanzaron diferencias en las medianas del grupo experimental al acabar el programa DTP $(M d n(\mathrm{y})=14,00 ; \mathrm{Z}=-1,941 ; p$ >.001). Estos resultados se pueden explicar en razón a la interacción individual que desarrollaron los sujetos en muchas actividades del programa. Aunque, los estudiantes sabían de su pertenencia al grupo en el cual trabajaban constantemente, estaban convencidos de su potencial individualista, lo cual no es claro en propuestas artivistas obtenidas de otros estudios que solo remiten mejoras a nivel humanístico y social (Elliott, Silverman y Bowman, 2016; Jivkova, Aladro y Sosa, 2019; Macaya y Valero, 2019). Aquí ha intervenido el contexto en el cual se desarrollaban los participantes (Ventanilla), del cual adoptaron formas conductuales de superación individualistas, ya que muchos de ellos eran sujetos que laboraban en contextos laborables cercanos a ellos, como también muchos de ellos apoyaban a sus padres, quienes eran comerciantes o negociantes en el mismo contexto de vulnerabilidad en que se encontraban.

\section{Conclusiones}

El objetivo general del estudio permitió descubrir los efectos del artivismo en la convivencia escolar y sus aprendizajes cooperativos, demostrándose que dicho artivis mo contextualizado en artivismo popular, como también la DTP, desarrollan el sentido de democracia y cooperación para convivencias en contextos en vulnerabilidad escolar. En esencia, el artivismo contextualizado en artivismo popular ha desarrollado aspectos estructurantes de la coexistencia como una necesidad de contextos sociales de primera atención. En principio, la pedagogía artivista popular ha desarrollado la convivencia democrática de modo significativo, al incluir contenidos coyunturales (problemáticos) de la propia sociedad para desarrollar la convivencia democrática en escolares que provenían de territorios con otras variables acompañantes como: pobreza social y pobreza económica. 
La colaboración y tolerancia, normativas para la convivencia y la resolución de conflictos como aspectos dimensionales de la convivencia democrática se desarrollaron mediante el ejercicio de actividades pedagógicas experimentales basadas en el artivismo popular. Los significados culturales, tanto folclóricos como ciudadanos permitieron introducir problemáticas con las cuales los escolares intervinieron a comunidades vulnerables que vivían con problemas de alto arraigo, y de cuyos contextos la escuela fue participe. Los escolares aprendieron a coexistir democráticamente con el fin de expresarse ante la sociedad vulnerable a la cual pertenecían.

En cuanto al aprendizaje cooperativo, este ha reaccionado como un complemento pedagógico de la experiencia artivista, ya que la DTP (dáctilo pintura teatral), introdujo a los escolares al escenario sensorial de la sociedad desde su conciencia corporal, pero, sobre todo, desde la conciencia mental. Los efectos significativos de esta propuesta en el grupo experimental, se establecieron en menor medida que el artivismo popular, pero con claros avances en dimensiones como la responsabilidad individual y grupal, interacción cara a cara, y las habilidades para socializar. La inmersión de los estudiantes en temáticas relativas al entorno permitió encontrar significativas relativas a los promedios o medianas más elevadas en la medición postest, pero con mayor énfasis en la capacidad para interactuar cooperando.

Los resultados en cuanto a la dimensión interacción positiva, demostraron la necesidad de replicar el estudio en contextos similares para comprobar se desarrolla de forma clara en sujetos con características y condiciones similares desde estrategias con DTP basadas en artivis mo social.

\section{Referencias}

Andrade, E. y Duran, G. (2017). Vivir el arte plástico y visual en escuela. Madrid, España: Estela Andrade.

Aladro-Vico, E.; Jivkova-Semova, D. y Baley, O. (2018). A new educative language for transformative social action. Comunicar, 26(57), 9 -18. https://doi.org/10.3916/C572018-01

Altun, S. (2015). The effect of cooperative learning on students' achievement and views on the science and technology course. International Electronic Journal of Elementary 
Education, $\quad 7(3), \quad 451-468 . \quad$ Recuperado de https://www.iejee.com/index.php/IEJEE/article/view/91

Aran-Ramspott, S.; Fedele, M. y Tarrago, A. (2018). Youtubers' social functions and their influence on pre-adolescence, Comunicar, 26(57), 71 - 80. https://doi.org/10.3916/C57-2018-07

Arguello, M. (2016). Arte y psicomotricidad infantil. Ibarra, Ecuador: Edición Digital Universidad Técnica del Norte.

Baena, G. (2018). Metodología de la investigación. Serie integral por competencias. México D. F, México: Grupo Editorial Patria.

Bandura, A. (1979). Aprendizaje social y desarrollo de la personalidad. Madrid, España: Alianza Editorial.

Bernárdez, A.; Padilla, G. y Sosa, R. (2019). From action art to artivism on Instagram: Relocation and instantaneity for a new geography of protest. Catalan Journal of Communication y Cultural Studies, 11(2), 23-37. Doi: https://doi.org/10.1386/cjcs.11.1.23_1

Bradshaw D. (2016). Art Integration Fosters Empathy in the Middle School Classroom. The clearing house: A journal of educational strategies, issues and ideas, 89(4-5), 109-117, doi https://doi.org/10.1080/00098655.2016.1170441

Carrillo, E.; Civís, M.; Andrés, T.A.; Longás, E. y Riera, J. (2018). Condicionantes del éxito y fracaso escolar en contextos de bajo nivel socioeconómico. Revista de estudios y $\begin{array}{llll}\text { experiencias en } & \text { educación, } & \text { 75-94, }\end{array}$ https://doi.org/10.21703/rexe.Especial2_201875944

Chib, A.; Bentley, C. y Wardoyo, R.J. (2019). Entornos digitales distribuidos y aprendizaje: Empoderamiento personal y transformación social en colectivos discriminados. Comunicar, 58(XXVII), 51-61, https://doi.org/10.3916/C58-2019-05

Chikh, A. y Hank, S. (2016). Towards a cooperative learning approach using intellige nce based learners grouping. Computer Applications in Engineering Education, 24(4), 639650. https://doi.org/10.1002/cae.21739

Colcord, C. R., Mathur, S. P. y Zucker, S. H. (2016). Improving disciplinary practices in an urban school: Solving the problem of practice. Journal of Education and Training Studies, 4(10), 144-155. doi: https://doi.org/10.11114/jets.v4i10.1811 
Elliott, D.; Silverman, M. y Bowman, W. (2016). Ciudadanía artística: arte, responsabilidad social y praxis ética. Estados Unidos: Oxford University Press.

García, J.J.; Parada, N.J. y Ossa, A.F. (2017). El drama creativo una herramienta para la formación cognitiva, afectiva, social y académica de estudiantes y docentes. Revista Latinoamericana de Ciencias Sociales, Niñez y Juventud, 15(2), 839-859. https://www.redalyc.org/articulo.oa?id=773/77352074005

Gass, M.; Gough, S.; Armas, A. y Dolcino, C. (2016). Play for Peace as a Violence Prevention Model: Achieving Voluntad y Convivencia. Journal of Experiential Education, 39(4), 412-426. doi: https $/ /$ doi.org/10.1177/1053825916674978

Gelabert, G. (2017). La práctica del canto colectivo como eje transversal de conocimientos, actitudes y valores: una propuesta dirigida a alumnos de Grado en Educación Infantil y Primaria. Foro de Educación, 15 (22), 1-21, http://dx.doi.org/10.14516/fde.505

Gestión, Zona Lounge (30 de setiembre de 2019). [FOTOS] Arte urbano en Lima: un recorrido por sus obras. Recuperado de: https://gestion.pe/especial/zonalounge/arte-y$\underline{\text { diseno/fotos-arte-urbano-lima-conozca-sus-obras-emblematicas-noticia-1994643 }}$

Grau, R. y García-Raga, L. (2017a). Learning to live together: a challenge for schools located in contexts of social vulnerability. Journal of Peace Education, 14, 1-18. Doi: https://doi.org/10.1080/17400201.2017.1291417

Grau, R. y García-Raga, L. (2017b). ConVivim: aplicación de un programa para aprender a convivir democráticamente. Revista electrónica de investigación educativa, 20(2), 8092. Recuperado de: https://dialnet.unirioja.es/ejemplar/489976

Grau, R.; García-Raga, L. y López-Martín, R. (2016). Towards School Transformation. Evaluation of a Coexistence Program from the Voice of Students and Teachers. Journal of New Approaches in Educational Research, 5(2), 137-146. doi: https://doi.org/10.7821/naer.2016.7.177

Greene, P. (2018). The Play's the Thing: Experimentally Examining the Social and Cognitive Effects of School Field Trips to Live Theater Performances. Educational Researcher, 23(6) , 1 -9, https $/ /$ doi.org/10.3102/0013189X18761034

Groos, K. (2012). The play of man. New York, USA: Good Press. 
Guillén, D. (26 de abril de 2017). El arte en la educación. Es educativo e instructivo frente a la enseñanza del arte. Recuperado de: https://pablogui.blogspot.com/2017/04/elarte-popular-peruano.html

Hernández, A.A.; Ramos, M.P.; Placencia, B.M.; Indacochea, B.; Quimis, A.J. y Moreno, L.A. (2018). Metodología de la investigación científica. Alicante, España: 3Ciencias, Área de Innovación y Desarrollo.

Huang, M. Y.; Tu, H. Y.; Wang, W. Y.; Chen, J-F.; Yu, Y. T. y Chou, C. C. (2017). Effects of cooperative learning and concept mapping intervention on critical thinking and basketball skills in elementary school. Thinking Skills and Creativity, 23, 207-216. https://doi.org/10.1016/j.tsc.2017.01.002

Jivkova, D.; Aladro, E. y Sosa, R. (2019). Entender el artivismo. Suiza: Peter Lang UK.

Kim, H. (2015). Community and art: creative education fostering resilience through art. Asia Pacific Education Review, 16 (2), 193-201, https://doi.org/10.1007/s12564-015-9371$\underline{Z}$

Laberintosurburbanos (6 de setiembre de 2015). Cómicos ambulantes limeños. Recuperado de: https://laberintosuburbanos.wordpress.com/tag/comicos-ambulantes-limenos/

Loughlin, S. (2015). "Reading" Paintings: Evidence for Trans-Symbolic and SymbolSpecific Comprehension Processes. Cognition and Instruction, 33(3), 257-293, https://doi.org/10.1080/07370008.2015.1076822

Macaya, A. y Valero, E. (2019). Proyecto Retrato Social: lo que la educación formal puede aprender del arte comunitario. Arte, individuo y sociedad, 31(1), 165-182. doi: http://dx.doi.org/10.5209/ARIS.60086

Maslow, A. (1991). Motivation and personality. Madrid, España: Harper y Row.

Mayordomo, R. y Onrubia, J. (2015). El aprendizaje cooperativo. Barcelona: Oberta UOC.

Mesías-Lema, J.M. (2018). Artivismo y compromiso social: transformación la educación del profesorado desde la sensibilidad. Comunicar, 57, 19-28. Doi: https://doi.org/10.3916/C57-2018-02

Ministerio de Educación del Perú - Minedu (2018). Número de casos reportados en el Síseve a nivel nacional (www.siseve.pe) del 15/09/2013 al 31/12/2018. Recuperado de: http://www.siseve.pe/web/ 
Ministerio de Educación del Perú (Minedu, 2019). Número de casos reportados en el Síseve a nivel nacional (www.siseve.pe) del 15/09/2013 al 31/12/2019. Recuperado de: http://www.siseve.pe/web/

Mélich, J.C. (1996). Antropología simbólica y acción educativa. Barcelona: Paidós.

Morales, M. y López, V. (2018). Políticas de convivencia escolar en América Latina: Cuatro Perspectivas de Comprensión y Acción. Archivos Analíticos de Políticas Educativas, 27(5). http://dx.doi.org/10.14507/epaa.27.3800

Niemann, C. (2005). La construcción social de la realidad según Peter L. Berger y Thomas Luckmann. Buenos Aires, Argentina: Amorrortu editores.

Proyecto Educativo Metropolitano (PEM, 2014). Lima Ciudad Educadora, amiga de los niños y las niñas. Lima, Perú: Municipalidad Metropolitana de Lima.

Prova, A. (2017). La práctica del aprendizaje cooperativo: Propuestas operativas para el grupo-clase. Madrid, España: Narcea ediciones.

Publimetro.pe (28 de diciembre de 2018). ¿Por qué les gusta tanto a los peruanos los cómicos ambulantes?. Recuperado de: https://publimetro.pe/actualidad/que-les-gusta$\underline{\text { tanto-peruanos-comicos-ambulantes-92792-noticia/?ref=pur }}$

Redford, J.; Desrochers, D. y Hoyer, K. M. (2017). The Years before School: Children's Nonparental Care Arrangements from 2001 to 2012. Stats in Brief. NCES 2017-096. National Center for Education Statistics. Recuperado de https://files.eric.ed.gov/fulltext/ED573320.pdf

Rogers, C. R. (1961). On becoming a person: A therapists's view of psychotherapy. Boston, USA: Houghton Mifflin.

Ruiz, M. (2014). Materiales, elementos y técnicas para el desarrollo de la expresión artístico plástica en el currículo de educación infantil. Innovación y experiencias educativas, 1(49), 323-346. Recuperado de: https://www.csif.es/contenido/sevilla/genera1/243960

Samah, A. (2016), Digital Tools: Enhancing Painting Skills among Malaysian Secondary School Students. The Turkish Online Journal of Educational Technology, 15(3), 124132, http $/ /$ www.tojet.net/

Spy Urban Art (2013). Ellos. Recuperado de: http //spy-urbanart.com/work/ellos/

Tello, A.F. y Obando, O.L. (2019). Discursos sobre el arte callejero como acción participativa desde las voces de jóvenes artistas callejeros (tesis de grado), 
Universidad del Valle, Santiago de Cali, Colombia. Recuperado de: http://bibliotecadigital.univalle.edu.co/handle/10893/12980

Tirado, R. y Conde, S. (2016). Análisis estructural de la gestión de la convivencia escolar en centros de buenas prácticas de Andalucía (España). Educación XX1, 19(2), 153-178. http://dx.doi.org/10.5944/educXX1.16459

Uriz, M. y Esteve-Mon, F. (2016). Dispositivos móviles y de aprendizaje cooperativo: Diseño de una intervención con dispositivos móviles en un entorno de aprendizaje cooperativo en la etapa de Educación Primaria. Edutec. Revista Electrónica de Tecnología Educativa, (58), 350. https://doi.org/10.21556/edutec.2016.58.833

Van, T.; Quintero, A. y Pizzutto, E. (2018). "Vamos a la Escuelita Arte Terapia": An Art Therapy Protocol for Promoting Resilience with Latino Farm Worker Children. Journal of Infant, Child y Adolescent Psychotherapy, 17(3), 213-228. http://dx.doi.org/10.1080/15289168.2018.1490068

Uberman, A. (1998). The Use of Games for Vocabulary Presentation and Revision. Forum, 36(1). https://eric.ed.gov/?id=EJ595092

Valdés, R.; López, V. y Chaparro, A.A. (2018). Convivencia escolar: adaptación y validación de un instrumento mexicano en Chile. Revista Electrónica de Investigación Educativa, 20(3), 80-91. https://doi.org/10.24320/redie.2018.20.3.1720

Vigotsky, L. S. (1978). Mind in society: The development of higher psychological processes. Massachusetts, Estados Unidos: Harvard University Press. 